Check for updates

Cite this: RSC Adv., 2018, 8, 36571

\title{
In situ and controllable synthesis of Ag NPs in tannic acid-based hyperbranched waterborne polyurethanes to prepare antibacterial polyurethanes/Ag NPs composites
}

\begin{abstract}
Shun Luo, $\dagger^{\mathrm{a}}$ Lin Fan, $\dagger^{\mathrm{b}}$ Kai Yang, ${ }^{\mathrm{a}}$ Zhen Zhong, ${ }^{a}$ Xiaojian $\mathrm{Wu}^{\mathrm{a}}$ and Tianbin Ren (D) *a
In this research, a simple and facile method was developed for preparation of antibacterial polyurethanes/ Ag NPs composites, where tannic acid-based hyperbranched waterborne polyurethanes (THWPU) was employed as both reductant and stabilizer to in situ and controllably synthesize Ag NPs at mild room temperature. The resultant Ag NPs in THWPU was confirmed by UV spectrophotometer, SEM and EDX. The effects of reaction temperature on the properties of the formed Ag NPs were investigated, and the results showed that the formed Ag NPs under room temperature and $80^{\circ} \mathrm{C}$ were both spherical, whereas increasing the reaction temperature benefits for promoting the formation of $\mathrm{Ag}$ NPs and narrowing the size distribution of the formed Ag NPs. Furthermore, triangular Ag NPs were in situ synthesized in THWPU via adding strong reductant $\left(\mathrm{NaBH}_{4}\right)$ during the formation of $\mathrm{Ag}$ NPs. Both spherical and triangular Ag NPs enhanced the mechanical property, thermal stability and antibacterial performance of THWPU. More importantly, our results demonstrated that formation of spherical Ag NPs in THWPU were beneficial for improving the mechanical property and thermal stability of THWPU, while the formation of triangular Ag NPs enabled THWPU better antibacterial performance against $E$. coli and S. aureus. Given these advantages, our obtained Ag NPs nanocomposites could be suitable for a broad range of commercial applications, such as medical products, children's products.
\end{abstract}

Received 11th September 2018 Accepted 15th October 2018

DOI: $10.1039 / c 8 r a 07575 a$

rsc.li/rsc-advances develop simple and effective techniques to fabricate highperformance WPUs/Ag NPs composites equipped with outstanding thermal, mechanical and antibacterial properties.

Recently, an alternative and promising strategy for the preparation of WPUs/Ag NPs composites is in situ synthesis of $\mathrm{Ag}$ NPs using WPUs as a stabilizing agent. ${ }^{9-11}$ The formed $\mathrm{Ag}$ NPs were demonstrated the homogeneous distribution in the WPUs matrix and displayed excellent stability without any agglomeration. ${ }^{12}$ For example, an anionic WPU with carboxylate-functional group was tailored synthesized via the introduction of bis (hydroxymethyl) propionic acid (DMPA) in the backbone of WPU and was employed as a stabilizing agent to in situ form Ag NPs for preparation of WPUs/Ag NPs composites. The prepared WPUs/Ag NPs composite films displayed improved thermal stability, mechanical performance and water resistance property as well as excellent antibacterial behavior against $E$. coli. ${ }^{13-15}$ Benefiting from these charming attributes, various WPUs have been synthesized and served as stabilizing agents to in situ synthesize Ag NPs in the WPUs matrix. ${ }^{15,16}$ Despite the fruitful research conducted on the preparation of WPUs/Ag NPs composites, the majority of the used WPUs were used as stabilizers and limited WPUs as both reductant and stabilizer for in situ formation of Ag NPs have been reported. ${ }^{14}$ In addition, the properties of the formed $\mathrm{Ag}$
${ }^{a}$ Institute of Nano and Biopolymeric Materials, School of Materials Science and Engineering, Key Laboratory of Advanced Civil Engineering Materials, Ministry of Education, Tongji University, 4800 Caoan Road, Shanghai 201804, China. E-mail: rentianbin008@163.com; Fax: +86-21-33515906; Tel: +86-21-33515906

${ }^{b}$ Department of Orthopedics, Shanghai Tenth People's Hospital, Tongji University School of Medicine, Shanghai, China

$\dagger$ These two authors contribute equally to this paper. 
NPs in WPUs matrix including size and shape play critical roles in the thermal stability, mechanical performance, water resistance property and antibacterial behaviour of the obtained WPUs/Ag NPs composites. Regrettably, there are few studies to tune and control the properties of the formed Ag NPs in WPUs matrix during the in situ synthesis process of Ag NPs. ${ }^{17}$

Herein, a tannic acid (TA)-based hyperbranched WPU (THWPU) was synthesized by using TA as a bio-based component and then was used to synthesize Ag NPs as both reductant and stabilizer. The effects of reaction time and temperature on the size and size distribution of the formed Ag NPs were investigated. The control method for the shape of Ag NPs formed in the THWPU was studied. Furthermore, the stability, mechanical properties and antibacterial properties of the prepared THWPU/Ag NPs composites with different shapes of Ag NPs were evaluated.

\section{Experimental}

\section{Materials}

Poly(tetramethylene ether glycol) (PTMG, $M_{\mathrm{n}}=2000$ ) and isophorone diisocyanate (IPDI) were obtained from Jining Baichuan Chemical Co., Ltd, China. Before using, PTMG-2000 was placed in a vacuum oven at $110{ }^{\circ} \mathrm{C}$ for $2 \mathrm{~h}$ to remove the moisture. Bis(hydroxymethyl)propionic acid (DMPA) was purchased from Shanghai Demand Chemical Co., Ltd, China. Dibutyltin dilaurate and stannous octoate were provided by Jiangsu Yoke Technology Co., Ltd, China. Triethylamine (TEA), tannic acid (TA), diethylene glycol (DEG), tetrahydrofuran (THF) and silver nitrate $\left(\mathrm{AgNO}_{3}\right)$ were obtained from Sinopharm Chemical Reagent Shanghai Co., Ltd., China and used as received. Escherichia coli (E. coli, ATCC 25922) and Staphylococcus aureus (S. aureus, ATCC29213) were obtained from Nanjing Bianzhen Biological Technology (China).

\section{Synthesis of THWPU}

To prepare THWPU, the prefabricated PTMG-2000 was placed in a $50 \mathrm{~mL}$ four-neck round-bottom flask under $65{ }^{\circ} \mathrm{C}$, followed by addition of IPDI. Then the mixture was heated to $80^{\circ} \mathrm{C}$ and kept for $2 \mathrm{~h}$ to prepare the -NCO-ended polyurethane prepolymer. After 2 h later, DMPA, DEG, dibutyltin dilaurate and stannous octoate were added for further reaction of $4 \mathrm{~h}$ to get the polyurethane prepolymer with $-\mathrm{COOH}$ groups. The mixture was cooled to $25^{\circ} \mathrm{C}$, and then the TA solution dissolved in THF was added, followed by keeping the reaction at $60{ }^{\circ} \mathrm{C}$ for $5 \mathrm{~h}$. After that, the mixture was placed in a water bath until the temperature dropped to $25{ }^{\circ} \mathrm{C}$, and was then neutralized by slowly adding TEA under stirring in $45 \mathrm{~min}$. The mixture was then vigorously stirred for $30 \mathrm{~min}$, followed by adding water for emulsification and vacuum evaporation to remove THF to obtain the waterborne polyurethane solution.

\section{In situ synthesis of Ag NPs with THWPU}

The THWPU solution with a solid content of $20 \%$ was obtained by dilution with deionized (DI) water, and then the silver nitrate solution of $0.1 \mathrm{~mol} \mathrm{~L}^{-1}$ was added into the solution under stirring and dark condition. The preparation process was illustrated in Scheme 1a. The ratio of $\mathrm{Ag}^{+}$to Benzene ring was 1/ 1.5. The effects of the reaction time and temperature on the size of the formed $\mathrm{Ag}$ NPs were investigated. Furthermore, to tune the shapes of the formed Ag NPs, sodium borohydride as a strong reductant was added into the mixture during the reaction process, as shown in Scheme $1 \mathrm{~b}$. The ratio of $\mathrm{Ag}^{+} /$ benzene ring/ $/ \mathrm{NaBH}_{4}$ was $1 / 1.5 / 1$.

\section{Antibacterial activity test of the THWPU/Ag NPs composites}

To quantitatively assess the antibacterial behaviours on the obtained THWPU/Ag NPs composite films, a shake flask method was used in this study following the test standard of ASTM E-2149 (2013). ${ }^{18}$ Typically, the film samples with $5 \times 5$ $\mathrm{cm}^{2}$ in size were used in this method. Nutrient broth was used as a growing medium for Gram-negative E. coli and Grampositive $S$. aureus, and phosphate buffered saline solution were chosen as a testing medium. In this work, the bacteria were cultivated in $5 \mathrm{~mL}$ of nutrient broth at $37^{\circ} \mathrm{C}$ for $24 \mathrm{~h}$. The film samples and initial suspended bacteria of $106 \mathrm{CFU} \mathrm{mL}^{-1}$ were placed into a $250 \mathrm{~mL}$ flask containing $70 \mathrm{~mL}$ of phosphate buffer saline. The flask was shaken on a reciprocal shaker at a speed of $220 \mathrm{rpm}$ at $37{ }^{\circ} \mathrm{C}$ for $1 \mathrm{~h}$. The plate count agar technique was then used for quantitative assessment of testing specimens. A 10-fold serial dilution technique was used for ensuring a reliable accounting of the bacterial colonies. Then $0.5 \mathrm{~mL}$ of bacterial suspension after shaking was placed over the agar into sterilized Petri dishes. Then bacteria colonies were counted after incubation at $37{ }^{\circ} \mathrm{C}$ for $48 \mathrm{~h}$. The antibacterial ratio was calculated as follows:

$$
\text { Antibacterial ratio }=\frac{N_{0}-N}{N_{0}} \times 100 \%
$$

where $N_{0}$ is the mean number of bacteria on the pristine THWPU film, and $N$ is the mean number of bacteria on the THWPU/Ag NPs composite film. This test was repeated three times for each sample.

\section{Characterization}

The particle size and size distribution of the prepared THWPU were measured by dynamic light scattering (DLS, Malvern ZEN3690) at $25{ }^{\circ} \mathrm{C}$. Three different samples were tested repeatedly. To evaluate the relative molecular weight and its distribution of the THWPU, gel permeation chromatography (GPC) spectrum was measured in THF with a concentration of $2 \mathrm{mg} \mathrm{mL}{ }^{-1}$. The flow rate and temperature were set at 1.0 $\mathrm{mL} \min ^{-1}$ and $40{ }^{\circ} \mathrm{C}$, respectively. UV-Vis absorption spectra of the THWPU/Ag NPs composite samples were recorded on a HITACHI U-1800 spectrophotometer at room temperature with a concentration of $0.1 \mathrm{mg} \mathrm{mL}^{-1}$. The X-ray diffraction (XRD) measurements were carried out by using a D/max-IIIA. Xray diffractometer (Rigaku Denki, Tokyo, Japan) with a $\mathrm{Cu}-\mathrm{K} \alpha$ radiation $(\lambda=0.154 \mathrm{~nm})$ at a scanning rate of $4^{\circ} \mathrm{min}^{-1}$. To observe the morphology of the THWPU/Ag NPs composite dispersion, a JEOL JEM-2100 transmission electron microscope (TEM) operating at an acceleration voltage of $120 \mathrm{KV}$ was 
(a)
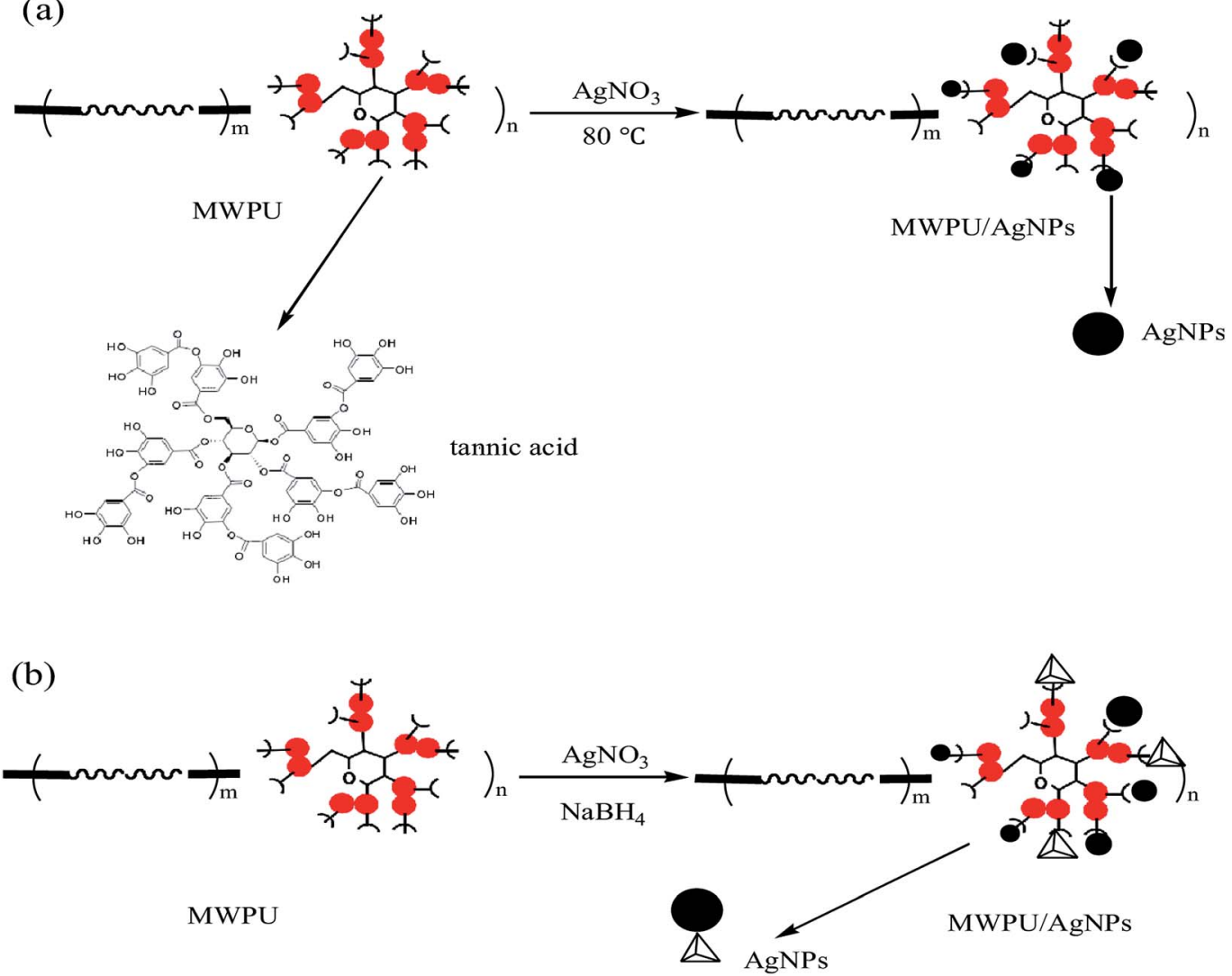

Scheme 1 Schematic diagram of the in situ synthesis process of Ag NPs within THWPU to prepare THWPU/Ag composites.

employed. The sample was prepared by dripping a droplet of a diluted THWPU/Ag NPs dispersion $\left(0.5 \mathrm{mg} \mathrm{mL}{ }^{-1}\right)$ onto a $450 \mathrm{~nm}$ carbon-coated copper grid and then dried at room temperature. The surface morphology of the THWPU/Ag NPs composite was observed by using a scanning electron microscopy (SEM, FEI, USA). The distribution of Ag element on the surface of THWPU/Ag NPs composite was determined via energy dispersive X-ray spectroscopy (EDX) using a Bruker X Flash Detector 6/30 instruments. To study the thermal stabilities of the THWPU and THWPU/Ag NPs composites, thermogravimetric analysis (TGA) was performed on TA Instruments Q500 (New Castle, DE, USA). The data was recorded from 20 to $800^{\circ} \mathrm{C}$ with a scanning rate of $20^{\circ} \mathrm{C} \mathrm{min}^{-1}$. The tensile strength, Young's modulus and elongation at break of the samples were studied by using a tensile tester (INSTRON5565, USA) at a speed of $50 \mathrm{~mm} \mathrm{~min}{ }^{-1}$ at room temperature. Three specimens were tested for each sample. The specimen was moulded into $45 \mathrm{~mm}$ long, $8 \mathrm{~mm}$ wide and $0.2 \mathrm{~mm}$ thick.

\section{Results and discussion}

\section{Characterization of THWPU}

The $20 \%$ wt THWPU solution exhibited yellow colour (Fig. 1a), which mainly derived from the TA's colour. The average size of THWPU determined by DLS was $100 \pm 5 \mathrm{~nm}$, with a narrow size distribution (PDI $<0.15$ ). The THWPU possess a negative surface potential, due to the presence of $-\mathrm{COOH}$ groups in the backbone of TA-based hyperbranched WPU. The GPC date shown in Fig. $1 \mathrm{~b}$ indicated that the molecular weight of the synthesized THWPU was $28600, M_{\mathrm{w}} / M_{\mathrm{n}}$ was 1.27 , and narrow molecular weight distribution of THWPU.

\section{In situ formation of Ag nanoparticles within THWPU}

In earlier work, TA have been demonstrated to reduce $\mathrm{Ag}^{+}$to $\mathrm{Ag}$ NPs via the reducibility of catechol groups. ${ }^{19}$ Inspired by these findings, THWPU was prepared and used to in situ synthesize Ag NPs as both reductant and stabilizer. As shown in Fig. 2a, after adding silver nitrate into THWPU solution, the colour of the mixture solution turned from yellowish to brown and did not form precipitation in the solution. The UV-Vis absorption spectrum was measured to confirm the generated Ag nanoparticles by tracking the characteristic absorbance peaks of the surface (a)

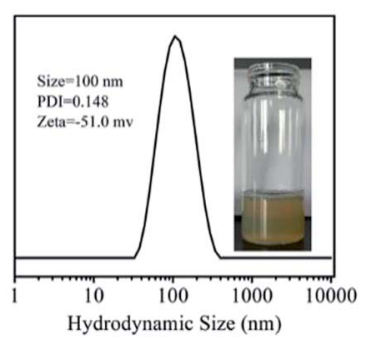

(b)

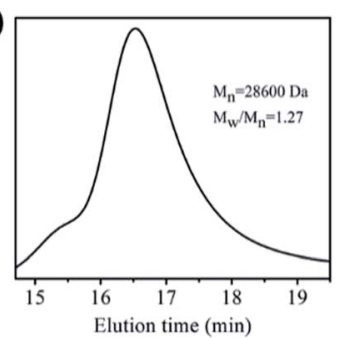

Fig. 1 The characterizations of THWPU by (a) DLS and (b) GPC analyses. 
(a)

政

(c)
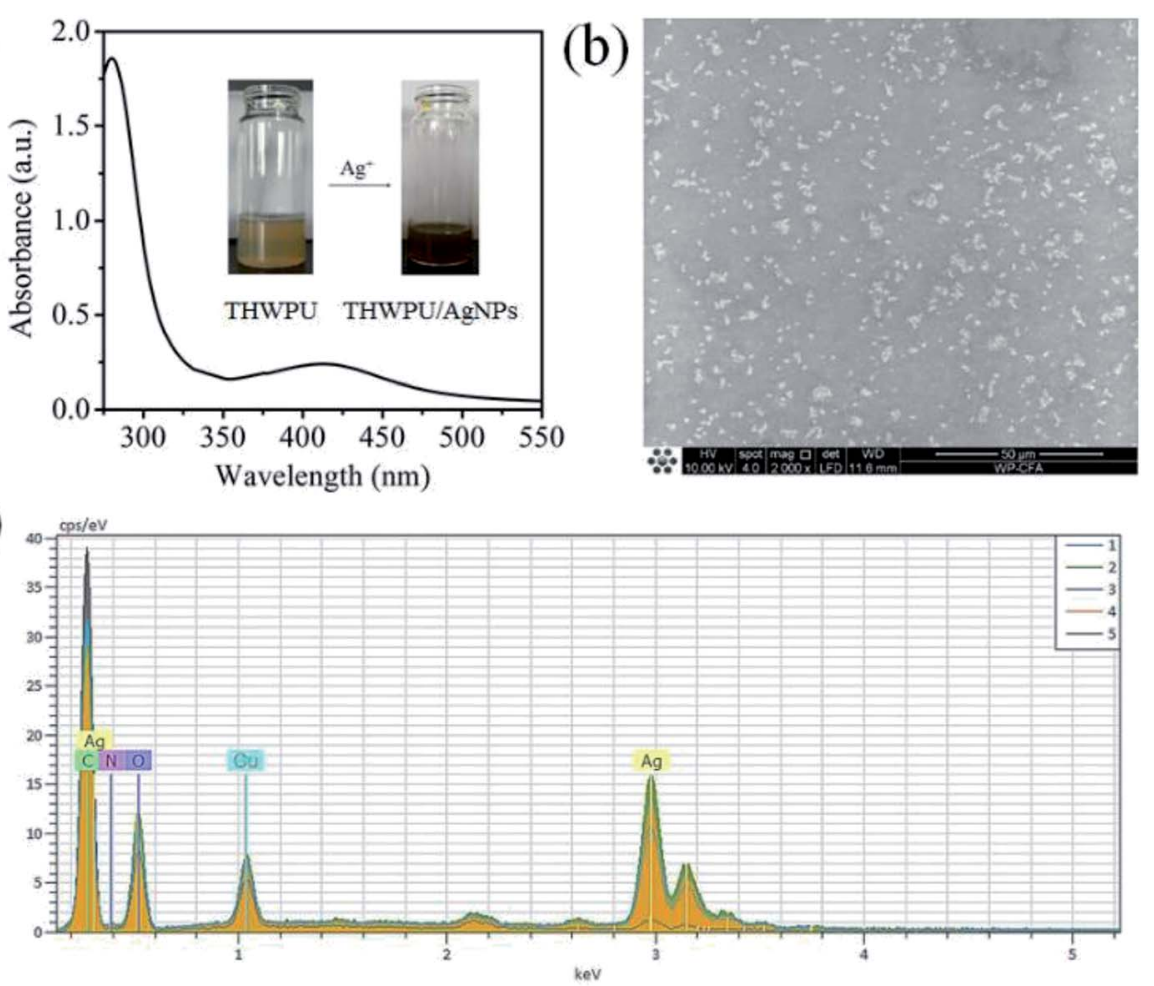

Fig. 2 (a)The UV-Vis spectrum and digital photos of THWPU/Ag NPs composites. (b) The SEM micrographs and (c) the EDX composition analysis of the THWPU/Ag NPs composite film.

plasmon resonance of Ag NPs at around $415 \mathrm{~nm} .{ }^{20}$ As shown in Fig. 2a, distinct characteristic absorption peak appeared at $418 \mathrm{~nm}$, showing the formation of Ag NPs within the THWPU.

To further affirm the formation of Ag NPs, the surface morphology of the THWPU/Ag NPs composite film prepared on a carbon-coated copper grid was observed with SEM. As shown in Fig. 2 b, densely populated nanoparticles were clearly observed in the film. The element composition of the observed nanoparticle was further analysed using energy dispersive X-ray spectroscopy (EDX). As illustrated in Fig. 2c, a strong characteristic peak of Ag appeared in the EDX spectrum, demonstrating that the nanoparticles observed in SEM images (Fig. 2b) were composed of silver. Furthermore, the characteristic peak of $\mathrm{Cu}$ was date from the copper grid, and the peak of $\mathrm{C}$ was date from the carbon support film on the copper grid or the THWPU. The appearance of peaks of $\mathrm{N}$ and $\mathrm{O}$ also verified the existence of THWPU which served as a protective layer of the formed Ag NPs. The mechanism of the formation of Ag NPs is that the residual catechol groups in THWPU could reduce $\mathrm{Ag}^{+}$to $\mathrm{Ag}^{0}$ by giving electrons to $\mathrm{Ag}^{+}$and conversing to quinone groups, the formed $\mathrm{Ag}^{\mathrm{O}}$ would be assembled to uniform $\mathrm{Ag}$ NPs due to the restriction of the hyperbranched structures in WPU. The aggregated Ag NPs could be fixed in the THWPU structure and be well protected by the hyperbranched WPU, thus demonstrate excellent stability.

The effects of reaction time and temperature on the generation of Ag NPs

The reaction time was critical to the formation of $\mathrm{Ag}$ NPs in THWPU. UV-Vis absorption spectra of formed Ag NPs with different reaction time were shown in Fig. 3a, the absorption peaks of $\mathrm{Ag}$ NPs become stronger as the reaction time increasing, indicating the population of the formed Ag NPs in THWPU rising. In addition, the peak position of the formed Ag NPs exhibited a redshift as reaction time prolonging, demonstrating the decreased size of the resulting Ag NPs. In addition, the absorption peak of Ag NPs obtained in this work ( $8 \mathrm{~h}$ ) was narrower compared with that of the reported Ag NPs prepared with anionic waterborne polyurethanes ${ }^{21}$ as templates, indicating the narrow size distribution of the formed Ag NPs in TAbased hyperbranched WPU.

Despite the fact that Ag NPs could be obtained with THWPU as reductant under mild room temperature, the synthesis process of Ag NPs is time-consuming due to the weak reducing capacity of catechol groups in THWPU. To enhance the reaction efficiency, the reaction temperature was adjusted to $80^{\circ} \mathrm{C}$. As shown in Fig. 3b, a more obvious absorption peak at $418 \mathrm{~nm}$ for $\mathrm{Ag}$ NPs was observed with the reaction time of $25 \mathrm{~min}$, indicating more Ag NPs were formed, compared with the spectrum
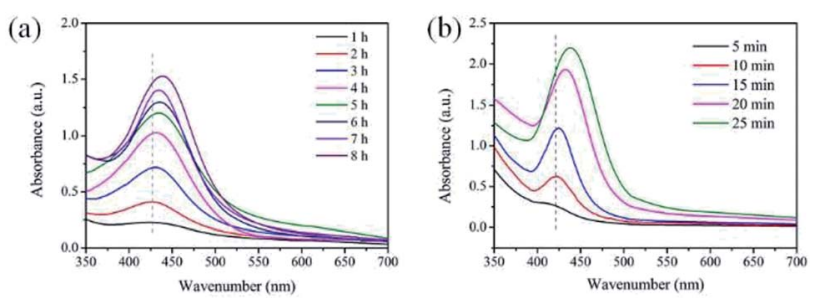

Fig. 3 The UV-Vis spectrum of THMPU/Ag NPs dispersions with various reaction time-points (a) at room temperature and (b) $80^{\circ} \mathrm{C}$. 
within $4 \mathrm{~h}$ under mild room temperature (shown in Fig. 3a). In addition, the absorption peak of $\mathrm{Ag}$ NPs formed at $80{ }^{\circ} \mathrm{C}$ was narrower than that of $\mathrm{Ag}$ NPs formed at room temperature, indicating that the generation of Ag NPs with narrower particle size distribution, which was profit from increasing reaction temperature.

The morphology of Ag NPs generated at $80{ }^{\circ} \mathrm{C}$ was further characterized by TEM. As shown in Fig. $4 \mathrm{a}$ and b, the Ag NPs were approximate sphere with a diameter of around 10-15 nm and evenly distributed in THWPU without agglomeration. The generated Ag NPs in this work exhibited the analogous shape, yet a smaller size and narrower size distribution, compared with those anionic waterborne polyurethanes as templates with $\mathrm{NaBH}_{4}$ as the reducing agent. ${ }^{10}$ Precisely because that the interior cavity of THWPU provides a moderate space for the
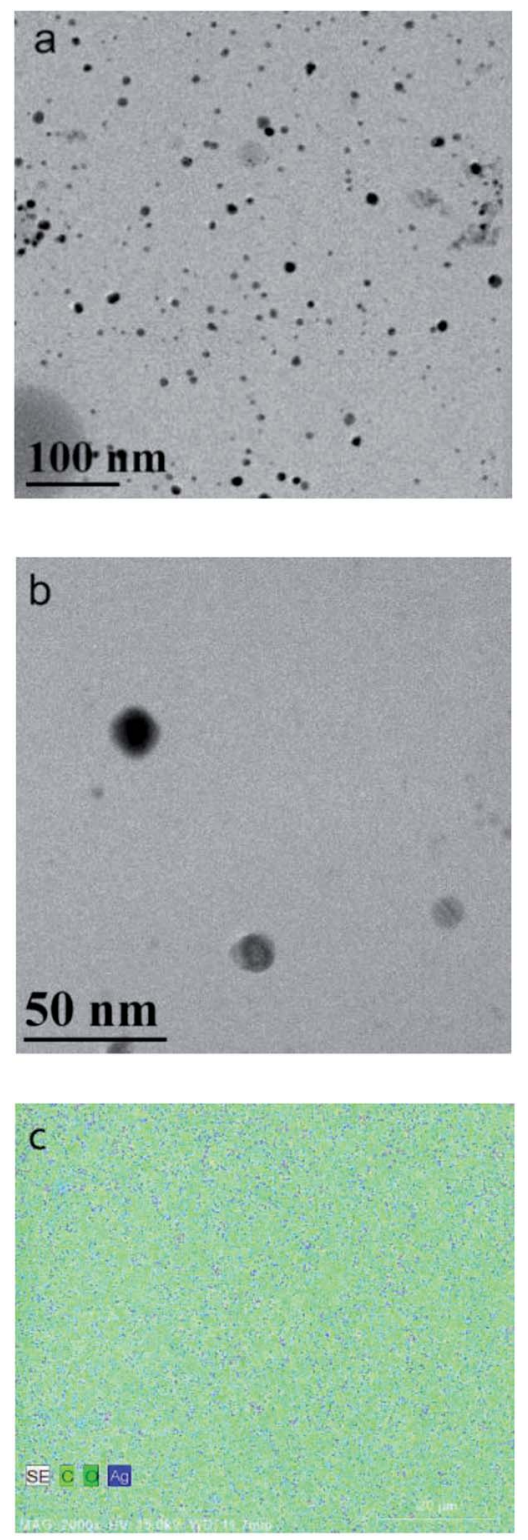

Fig. 4 ( $a$ and $b$ ) The TEM images of the formed Ag NPs in THWPU at a different scale and (c) EDX analysis of the THWPU/Ag NPs composite film.
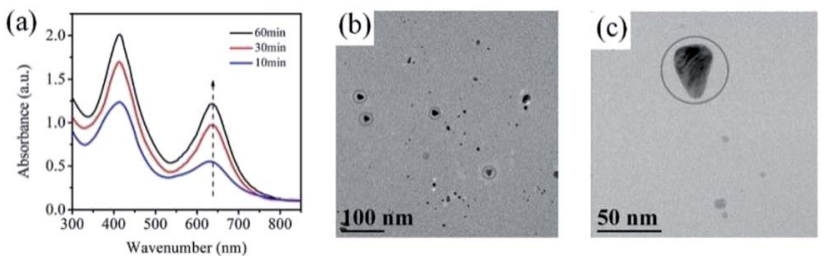

Fig. 5 (a) The UV-Vis absorption spectrum and ( $b$ and c) TEM images of THWPU/Ag NPs composites at a different scale using $\mathrm{NaBH}_{4}$ as reducing agent.

nucleation and germinate of the Ag NPs, which is favourable for the develop of smaller Ag NPs. Moreover, the protection layer of THWPU around the Ag NPs effectively suppressed the agglomeration of the formed Ag NPs, leading to a narrow size distribution. This result was further verified by the uniform distribution of $\mathrm{Ag}$ element in the THWPU/Ag NPs composite film, shown in Fig. 4c. While, TEM images shows Ag NPs very diluted, which indicates that the concentration of nano-silver is low.

\section{Preparation of Ag NPs with different shapes in THWPU}

Xia et al. reported the preparation of Ag NPs with different shapes through using Polyvinyl Pyrrolidone (PVP) as a protective agent. The shapes were regulated by adding various reducing agents at different growth stages of $\mathrm{Ag} \mathrm{NPs}^{.22}$ In this research, the effects of addition of a strong reductant $\left(\mathrm{NaBH}_{4}\right)$ in the mixed water solution of THWPU and $\mathrm{AgNO}_{3}$ on the

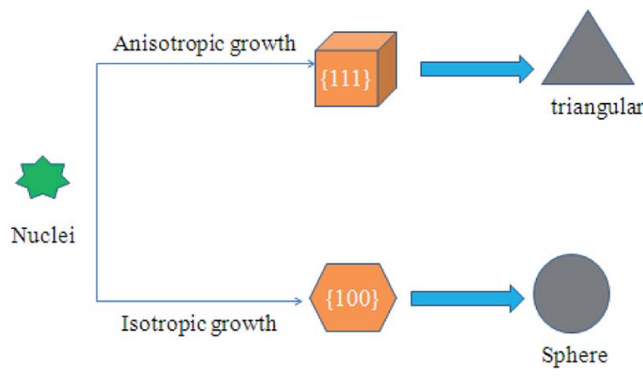

Scheme 2 Schematic diagram of Ag NPs growth mechanism using $\mathrm{NaBH}_{4}$ as reducing agent.

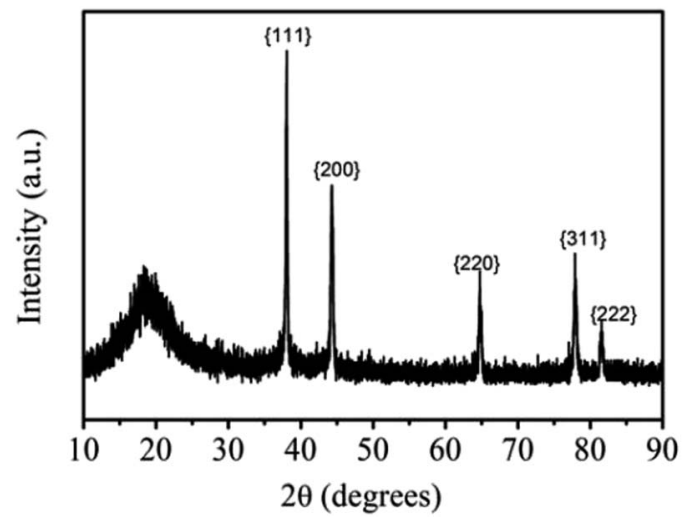

Fig. 6 The XRD spectrum of THWPU/Ag NPs composites by $\mathrm{NaBH}_{4}$. 

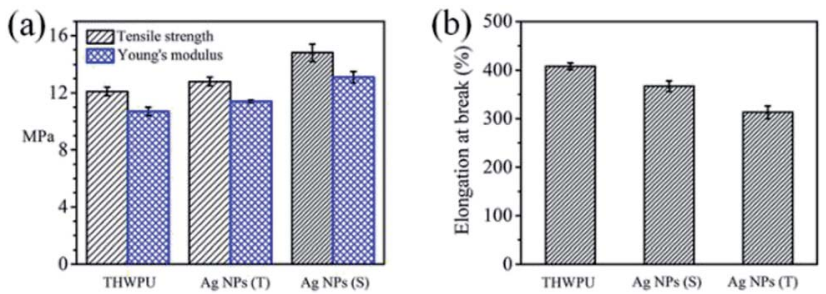

Fig. 7 The mechanical properties of THWPU, Ag NPs (T) and Ag NPs (S). (a) The tensile strength, Young's modulus and (b) elongation at breaks for different composites.

Table 1 The mechanical properties of THWPU, Ag NPs (T) and Ag NPs (S)

\begin{tabular}{llll}
\hline Samples & $\begin{array}{l}\text { Young's } \\
\text { modulus (MPa) }\end{array}$ & $\begin{array}{l}\text { Tensile strength } \\
(\mathrm{MPa})\end{array}$ & $\begin{array}{l}\text { Elongation at } \\
\text { break }(\%)\end{array}$ \\
\hline THWPU & $10.7 \pm 0.3$ & $12.1 \pm 0.3$ & $408 \pm 7$ \\
Ag NPs (S) & $13.1 \pm 0.4$ & $14.8 \pm 0.6$ & $367 \pm 11$ \\
Ag NPs (T) & $11.4 \pm 0.1$ & $12.8 \pm 0.3$ & $313 \pm 13$
\end{tabular}

growth of Ag NPs was investigated. The UV-Vis absorption spectra of the samples were recorded and shown in Fig. 5a. It could be clearly observed that there was a strong absorption peak at $630 \mathrm{~nm}$ and $420 \mathrm{~nm}$. According to Mie Scattering Theory (MST), small $\mathrm{Au}$ or $\mathrm{Ag}$ nanospheres have only one plasmon resonance absorption peak, and anisotropic Au or Ag nanoparticles could have 2 to 3 plasmonic absorption peaks depending on their shapes. ${ }^{23}$ The triangular Ag NPs possessed absorption peaks in the range of $600-700 \mathrm{~nm},{ }^{24}$ indicating that triangular Ag NPs may be generated in this system. The triangular morphologies of Ag NPs were further affirmed by TEM images (Fig. 5b and c).

The growth mechanism of nano-silver is not very clear up to now, growth mechanism of AgNPs is speculated in Scheme 2 . Typically, the growth process of Ag NPs can be divided into four phases: (1) $\mathrm{Ag}^{+}$is reduced triangular to $\mathrm{Ag}^{0}$; (2) $\mathrm{Ag}^{0}$ aggregates into crystal nucleus; (3) the nucleus develops to a stable seed crystal with a diameter of 2 to $6 \mathrm{~nm}$; (4) the stability of the system began to decline, the seed crystals further aggregate into lager stable nanoparticles. ${ }^{25}$

The THWPU prefers to interact with $\{111\}$ crystal planes of Ag NPs which have low surface energy, and therefore the crystals will grow along $\{100\}$ crystal planes to form spherical $\mathrm{Ag}$ nanoparticles in absence of the strong reductants such as (a)

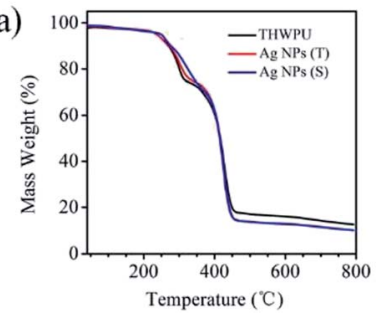

(b)

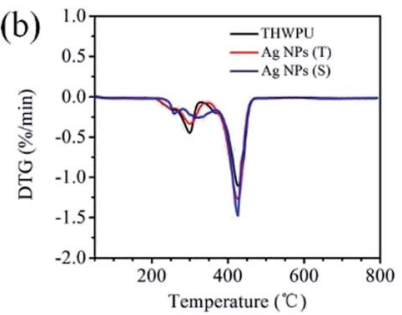

Fig. 8 (a) The TGA and (b) DTG profiles of THWPU, Ag NPs (S) and Ag NPs (T) composites film.
Table 2 The thermal decomposition temperatures of THWPU and Ag NPs composites with different shapes by thermal gravimetric analysis

\begin{tabular}{llllll}
\hline Samples & $T_{10 \%}\left({ }^{\circ} \mathrm{C}\right)$ & $T_{20 \%}\left({ }^{\circ} \mathrm{C}\right)$ & $T_{30 \%}\left({ }^{\circ} \mathrm{C}\right)$ & $T_{40 \%}\left({ }^{\circ} \mathrm{C}\right)$ & $T_{50 \%}\left({ }^{\circ} \mathrm{C}\right)$ \\
\hline THWPU & 269.50 & 300.93 & 365.44 & 401.17 & 413.25 \\
Ag NPs (T) & 272.17 & 307.79 & 367.11 & 401.90 & 413.76 \\
Ag NPs (S) & 276.56 & 311.65 & 376.75 & 401.40 & 414.52
\end{tabular}

$\mathrm{NaBH}_{4}$. Once $\mathrm{NaBH}_{4}$ is added, the formation of seed crystals of Ag NPs will be accelerated, and more Ag seed crystals will be formed within a brief period of time. In the meantime, the $\mathrm{pH}$ and thermodynamics of the system also get changed, making some seed crystals grow along the $\{111\}$ crystal planes and eventually grows into triangular Ag nanoparticles, as shown in Scheme 2.

As illustrated in the XRD pattern of the obtained the THWPU/Ag NPs composite film under addition of $\mathrm{NaBH}_{4}$ in Fig. 6, there is a strong diffraction peak at $38.11^{\circ}$ which is corresponding to the $\{111\}$ crystal planes of the Ag NPs, indicating the preferential orientation of $\{111\}$ crystal planes of Ag NPs and the growth mechanism of triangular Ag NPs along the $\{111\}$ crystal planes. In addition, the peaks at $20^{\circ}, 44.30^{\circ}, 64.4^{\circ}, 77.4^{\circ}$ and $81.54^{\circ}$ are corresponding to the crystallized soft segment of THWPU and $\{200\},\{220\},\{311\}$, and $\{222\}$ crystal planes of the Ag NPs, respectively. ${ }^{26}$

\section{The shape influences of the Ag NPs on the mechanical properties of the THWPU/Ag NPs composites}

To evaluate the mechanical properties of THWPU and THWPU/ Ag NPs composites, the tensile strength, Young's modulus and elongation at break were tested and corresponding results were shown in Fig. 7 and Table 1. The results show that both spherical Ag NPs in THWPU (Ag NPs (S)) and triangular Ag NPs in THWPU (Ag NPs (T)) could enhance the mechanical properties of THWPU. Compared with Ag NPs (S), Ag NPs (T) are worse to enhance the tensile strength and Young's modulus and maintain the flexibility of THWPU, probably due to the anisotropic stress discontinuity of triangular Ag NPs.

\section{The shape influences of the Ag NPs on the thermal properties of the THWPU/Ag NPs composites}

The thermal properties of the THWPU and its composites were characterized by TGA and DTG. As shown in Fig. 8 and Table 2, the $T_{20 \%}$ of THWPU/Ag NPs (S), THWPU/Ag NPs (T) and THWPU were $311.65{ }^{\circ} \mathrm{C}, 307.79{ }^{\circ} \mathrm{C}$ and $300.93{ }^{\circ} \mathrm{C}$, indicating both
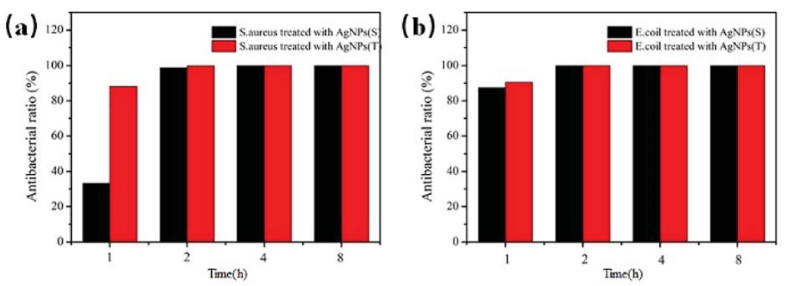

Fig. 9 The bacteriostatic ratios of Ag NPs composites with different shapes for Escherichia coli (a) and Staphylococcus aureus (b) at various time-points. 
Table 3 The bacteriostatic ratios of Ag NPs composites with different shapes for Escherichia coli and Staphylococcus aureus at various timepoints

\begin{tabular}{|c|c|c|c|c|c|c|}
\hline $1 \mathrm{~h}$ & $190 \times 10^{6}$ & $195 \times 10^{6}$ & $225 \times 10^{5} \pm 7$ & $180 \times 10^{5} \pm 24$ & $130 \times 10^{6} \pm 19$ & $245 \times 10^{5} \pm 13$ \\
\hline $4 \mathrm{~h}$ & $118 \times 10^{8}$ & $210 \times 10^{8}$ & None & None & $112 \times 10^{2} \pm 17$ & None \\
\hline $8 \mathrm{~h}$ & $200 \times 10^{9}$ & $195 \times 10^{9}$ & None & None & None & None \\
\hline
\end{tabular}

spherical and triangular $\mathrm{Ag}$ NPs could enhance the thermal stability of THWPU. The THWPU exhibited a three-stage thermal degradation process: (i) at the first stage, the decomposition of some thermolabile urethane bonds and ester bonds occurred at around $250{ }^{\circ} \mathrm{C}$; (ii) the second stage was corresponding to the decomposition of the aromatic rings from tannic acid (TA) at around $300{ }^{\circ} \mathrm{C}$; (iii) the third stage was at around $420^{\circ} \mathrm{C}$, involving the decomposition of the soft segment on the polyurethane backbone. The AgNPs that with different morphology can further improve the thermal stability of composites, while the main effect is reflected in the second stage of thermal decomposition of composites. And the improved thermal properties of the THWPU/Ag NPs composites were mainly caused by the interaction between Ag NPs and benzene rings in tannin acid.

\section{The shape influences of the Ag NPs on the antibacterial properties of the THWPU/Ag NPs composites}

The previous studies have demonstrated that the antibacterial properties of Ag NPs were highly dependent on their shape and anisotropic polyhedral. Ag NPs are reported to exhibit different bactericidal properties. Song et al. found that spherical Ag NPs and triangular flake-shaped Ag NPs are superior to rod-shaped $\mathrm{Ag}$ NPs in antibacterial property against E. coli. ${ }^{27}$ Herein, the antibacterial properties of the spherical and triangular Ag NPs in THWPU against $E$. coli and $S$. aureus were evaluated by the shake-flask vibration method. The inhibition rates of THWPU/ Ag NPs (S) and THWPU/Ag NPs (T) against E. coli and $S$. aureus at different incubation time are shown in Fig. 9 and Table 3. It could be observed that the inhibition rates of MWPU/ $\mathrm{Ag}$ NPs (T) against $E$. coli and $S$. aureus were $90.52 \%$ and $87.43 \%$
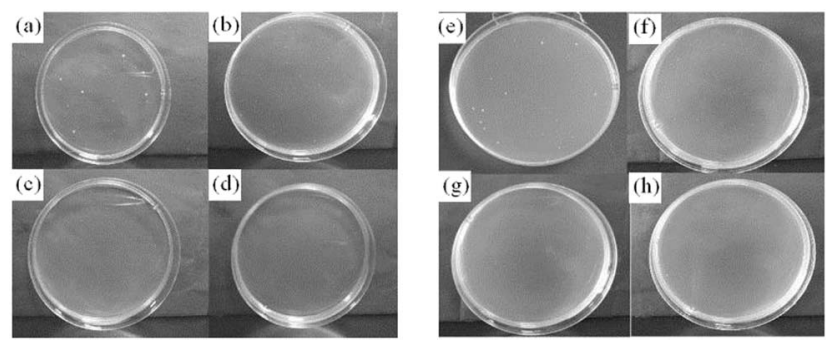

Fig. 10 The colony forming units (CFU) of Ag NPs composites with different shapes for Escherichia coli $(\mathrm{a}-\mathrm{d})$ and Staphylococcus aureus $(e-h)$ at various time-points. $(a-d)$ The colony forming units of Escherichia coli for $1 \mathrm{~h}, 2 \mathrm{~h}, 4 \mathrm{~h}$ and $8 \mathrm{~h}$ respectively. (e-h) The colony forming units of Staphylococcus aureus for $1 \mathrm{~h}, 2 \mathrm{~h}, 4 \mathrm{~h}$ and $8 \mathrm{~h}$ respectively. after shaking for $1 \mathrm{~h}$, while the inhibition rates of MWPU/Ag NPs (S) were $88.15 \%$ and $33.33 \%$ against $E$. coli and S. aureus, indicating better antibacterial properties of THWPU/Ag NPs (T). The better antibacterial ability of MWPU/Ag NPs (T) was probably due to the more $\{111\}$ crystal faces of the triangular Ag NPs which have a higher reactivity (Fig. 10). ${ }^{28}$

\section{Conclusions}

In this case, a simple and facile method was developed for preparation of antibacterial polyurethanes/Ag NPs composites, where tannic acid-based hyperbranched waterborne polyurethanes (THWPU) was employed as both reductant and stabilizer to in situ and controllably synthesize Ag NPs. The formation of Ag NPs within THWPU was demonstrated by UV spectrophotometer, SEM and EDX. The effects of reaction temperature on the formation of Ag NPs were investigated and the results showed that the formed $\mathrm{Ag}$ NPs under room temperature and $80{ }^{\circ} \mathrm{C}$ were both spherical, whereas the formation of Ag NPs at $80^{\circ} \mathrm{C}$ was much faster and the formed $\mathrm{Ag}$ NPs had narrower size distribution compared with those formed under room temperature. Furthermore, triangular $\mathrm{Ag}$ NPs were also synthesized in THWPU via adding strong reductant $\left(\mathrm{NaBH}_{4}\right)$ during the formation of Ag NPs. The mechanical property, thermal stability and antibacterial performance of the prepared THWPU/Ag NPs composites with different shapes were evaluated, and the results demonstrated that formation of spherical Ag NPs in THWPU were beneficial for improving the mechanical property and thermal stability of THWPU, while the formation of triangular Ag NPs enabled THWPU better antibacterial performance against $E$. coli and $S$. aureus. Given these advantages, our obtained Ag NPs nanocomposites could be suitable for a broad range of commercial applications, such as medical products, children's products.

\section{Conflicts of interest}

There are no conflicts to declare.

\section{Acknowledgements}

This work was financially supported by the National Natural Science Foundation of China (81571801, 81671944), the Fundamental Research Funds for the Central Universities and the Open Funds for Characterization of Tongji University. 


\section{References}

1 H. Q. Fu, Y. Wang, X. Y. Li and W. F. Chen, Compos. Sci. Technol., 2016, 126, 86-93.

2 S. H. Hsu, H. J. Tseng and Y. C. Lin, Biomaterials, 2010, 31, 6796-6808.

3 X. Q. Li, Y. J. Jiang, F. Wang, Z. J. Fan, H. N. Wang, C. H. Tao and Z. F. Wang, RSC Adv., 2017, 7, 46480-46485.

4 L. Q. Peng, L. C. Zhou, Y. F. Li, F. Pan and S. D. Zhang, Compos. Sci. Technol., 2011, 71, 1280-1285.

5 C. W. Chou, S. H. Hsu, H. Chang, S. M. Tseng and H. R. Lin, Polym. Degrad. Stab., 2006, 91, 1017-1024.

6 C. I. Wu, J. W. Huang, Y. L. Wen, S. B. Wen, Y. H. Shen and M. Y. Yeh, J. Chin. Chem. Soc., 2009, 56, 1231-1235.

7 H. W. Duan and S. M. Nie, J. Am. Chem. Soc., 2007, 129, 33333338.

8 Y. Y. Shen, G. J. He, Y. S. Guo, H. J. Xie and W. J. Fang, Nanoscale Res. Lett., 2017, 12, 525-533.

9 J. G. Han, Y. Q. Xiang and Y. Zhu, J. Inorg. Organomet. Polym., 2014, 24, 283-290.

10 Z. Zhong, S. Luo, K. Yang, X. J. Wu and T. B. Ren, RSC Adv., 2017, 7, 42296-42304.

11 W. S. Han, Polym. Compos., 2018, 39, 1967-1977.

12 N. Kutsevol, M. Bezuglyi, T. Bezugla and M. Rawiso, Mol. Cryst. Liq. Cryst., 2014, 590, 179-185.

13 F. Zhang, X. L. Wu, Y. Y. Chen and H. Lin, Fibers Polym., 2009, 10, 496-501.

14 Y. Wattanodorn, R. Jenkan, P. Atorngitjawat and S. Wirasate, Polym. Test., 2014, 40, 163-169.
15 H. Q. Fu, Y. Wang, X. Y. Li and W. F. Chen, Compos. Sci. Technol., 2016, 126, 86-93.

16 H. Liu, J. Song, S. B. Shang, Z. Q. Song and D. Wang, ACS Appl. Mater. Interfaces, 2012, 4, 2413-2419.

17 J. Helmlinger, C. Sengstock, C. Gross-Heitfeld, C. Mayer, T. A. Schildhauer, M. Koller and M. Epple, RSC Adv., 2016, 6, 18490-18501.

18 W. Pongnop, K. Sombatsompop, A. Kositchaiyong and N. Sombatsompop, J. Appl. Polym. Sci., 2011, 122, 3456-3465.

19 Y. Z. Cao, R. F. Zheng, X. H. Ji, H. Liu, R. G. Xie and W. S. Yang, Langmuir, 2014, 30, 3876-3882.

20 M. Hu, A. Ghoshal, M. Marquez and P. G. Kik, J. Phys. Chem. C, 2010, 114, 7509-7514.

21 B. Wiley, Y. G. Sun and Y. N. Xia, Acc. Chem. Res., 2007, 40, 1067-1076.

22 X. H. Xia, J. Zeng, Q. Zhang, C. H. Moran and Y. N. Xia, J. Phys. Chem. C, 2012, 116, 21647-21656.

23 S. K. Das, A. R. Das and A. K. Guha, Small, 2010, 6, 10121021.

24 Y. G. Sun and Y. N. Xia, Analyst, 2003, 128, 686-691.

25 J. Polte, X. Tuaev, M. Wuithschick, A. Fischer, A. F. Thuenemann, K. Rademann, et al., ACS Nano, 2012, 6, 5791-5802.

26 A. K. Sinha, M. Basu, M. Pradhan, S. Sarkar, Y. Negishi and T. Pal, Langmuir, 2011, 27, 11629-11635.

27 S. Pal, Y. K. Tak and J. M. Song, Appl. Environ. Microbiol., 2007, 73, 1712-1720.

28 S. Singh, A. Bharti and V. K. Meena, J. Mater. Sci.: Mater. Electron., 2015, 26, 3638-3648. 